\title{
Mitochondrial metabolism in cancer stem cells: a therapeutic target for colon cancer
}

\author{
In-Sung Song, Yu Jeong Jeong \& Jin Han* \\ National Research Laboratory for Mitochondrial Signaling, Department of Physiology, College of Medicine, Cardiovascular and Metabolic \\ Disease Center, Inje University, Busan 47392, Korea
}

\begin{abstract}
It has been proposed that the selective elimination of cancer stem cells (CSCs) using targeted therapy could greatly reduce tumor growth, recurrence, and metastasis. To develop effective therapeutic targets for CSC elimination, we aimed to define the properties of CSC mitochondria, and identify CSC-mitochondria-specific targets in colon cancer. We found that colon CSCs utilize mitochondrial oxidative phosphorylation (OXPHOS) to produce ATP. We also found that forkhead box protein 1 (FOXM1)-induced peroxiredoxin 3 (PRDX3) maintains the mitochondrial function, and the FOXM1/PRDX3 mitochondrial pathway maintains survival of colon CSCs. Furthermore, FOXM1 induces CD133 (PROM1/prominin 1) expression, which maintains the stemness of colon CSCs. Together, our findings indicate that FOXM1, PRDX3, and CD133 are potential therapeutic targets for the elimination of CSCs in colon cancer. [BMB Reports 2015; 48(10): 539-540]
\end{abstract}

Cancer stem cells (CSCs) are defined as a distinct subpopulation of cells within a tumor that have the ability to self-renew and differentiate. It has been proposed that CSCs, which are not destroyed by conventional treatments, are the cause of disease relapse and metastasis. Therefore, it is important for the identification and characterization of specific CSC targets for eliminating CSCs. Recently, it was reported to being the

*Corresponding author. E-mail: phyhanj@inje.ac.kr

http://dx.doi.org/10.5483/BMBRep.2015.48.10.179

Received 31 August 2015

Keywords: Colon cancer, Cancer stem cells, Peroxiredoxin 3, CD133, FOXM1

Abbreviations: CSC, Cancer stem cells; PRDX, Peroxiredoxin; ATP, adenosine triphosphate; FoxMl, forkhead box protein M1; qRT-PCR, quantitative reverse transcription-polymerase chain reaction; 5-FU, 5-fluorouracil; SCID, severe combined immunodeficiency; OXPHOS, Oxidative phosphorylation

Perspective to: Song et al. (2015), FoxM1-induced PRX3 Regulates Stemness and Survival of Colon Cancer Stem Cells via Maintenance of Mitochondrial Function, Gastroenterology, doi:10.1053/j.gastro. 2015.06.007. mitochondrial and energy metabolism-related properties in lung and ovarian CSCs. The studies focused on the differences in mitochondrial properties between CSCs and non-CSCs. However, the properties of CSC mitochondria involved in COlon cancer have not been clearly defined. Furthermore, there is a need to identify mitochondria-specific targets for the complete elimination of CSCs in colon cancer therapy. Based on this, we investigated how mitochondrial energy metabolism-related processes are regulated in CSCs. Aspects of mitochondrial function were assessed in colon CSCs, isolated from a colon cancer cell line, by fluorescence-activated cell sorting based on expression of CSC markers CD133, CD44, and Lgr5. As a result, we showed that CSC mitochondria have properties distinct from those of non-CSCs, as indicated by their increased mitochondrial $\mathrm{Ca}^{2+}$, membrane potential and reactive oxygen species (ROS) levels. The CSCs also had higher mitochondrial ATP levels and oxygen consumption rates than the non-CSCs. In addition, the CSC population increased under conditions of glucose deprivation and in low-glucose medium. Based on these data, we suggested that colon CSCs utilize mitochondrial oxidative phosphorylation (OXPHOS) to produce ATP, unlike the general cancer cells.

We determined that CSCs display enhanced resistance to an anticancer drug whose action is dependent on increased mitochondrial oxidative phosphorylation. With combination treatment of 5-fluorouracil (FU) and antimycin A, CSC death was induced as a result of antimycin A-mediated inhibition of mitochondrial complex III. Using quantitative RT-PCR and microarray analysis, we also found that the mitochondrial gene PRDX3 is highly upregulated in colon CSCs, compared with non-CSCs. In particular, qRT-PCR analysis showed increased PRDX3 expression in $\mathrm{CD}_{133^{+}}$CSCs freshly isolated from patients with colon cancer. Expression levels of PRDX3 and CD133 in CSCs isolated from patient tissues showed significant positive correlation. Moreover, immunofluorescence analysis showed co-localization of PRDX3 and CD133 in the tumor tissue. These data indicate that PRDX3 plays a critical role in mitochondrial function in CSCs and in colon carcinogenesis. To determine the role of PRDX3, we next used short interfering RNA (siRNA) and short hairpin RNA (shRNA) targeting the PRDX3 gene (siPRDX3 and shPRDX3, respectively) to deplete PRDX3 expression. In functional studies, siRNA- and 


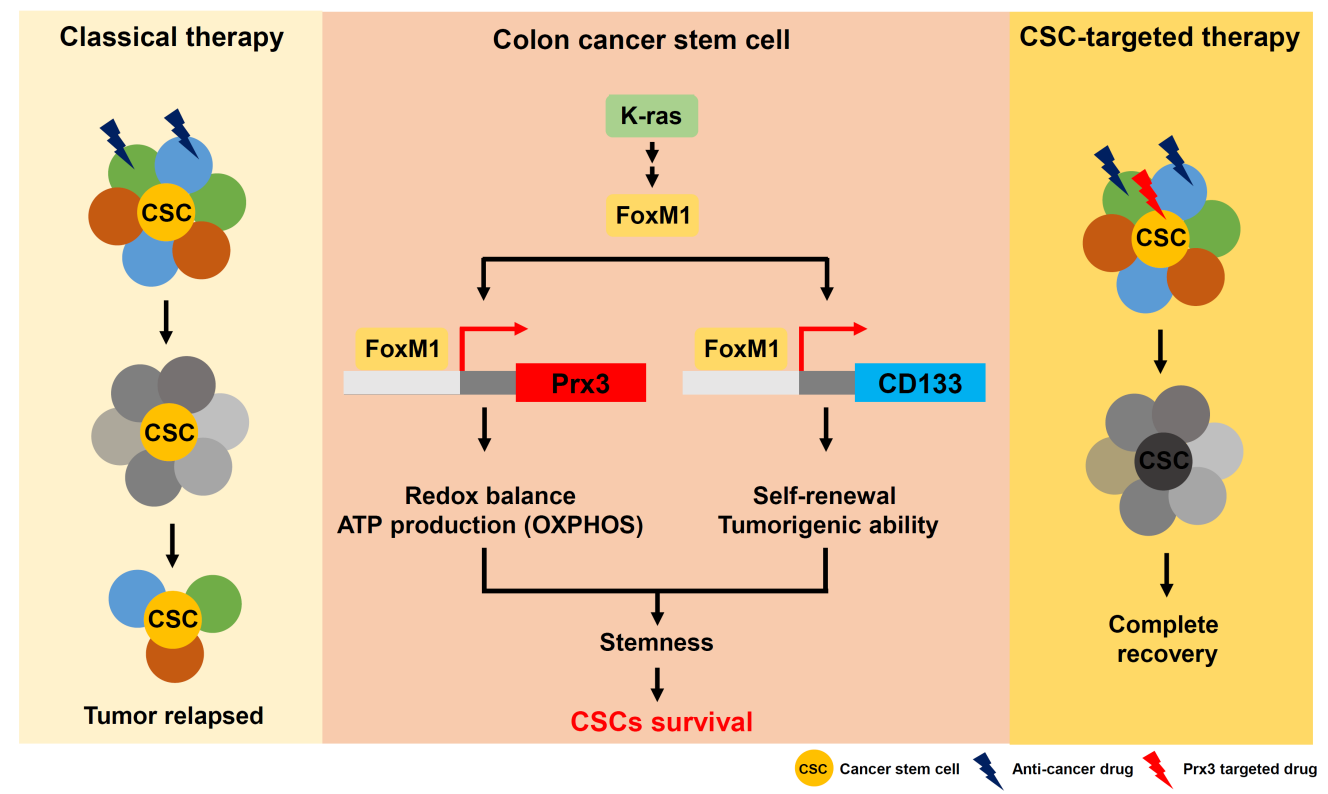

Fig. 1. A scheme outlining the therapeutic strategy of colon cancer and the regulating mechanism to stemness and survival of CSCs by Peroxiredoxin 3 and FoxM1 (Modified from Song et al (2015), Gastroenterology.

shRNA-mediated PRDX3 knockdown resulted in a significant decrease in the size of the CSC population, and sensitized the CSCs to 5-FU-induced cell death as a result of mitochondrial dysfunction. Furthermore, we used the xenograft and orthotopic xenograft models produced by the injection of $\mathrm{CD} 133^{+}$ cells (sorted from HT-29 human colorectal adenocarcinoma), PRDX3 knockdown (HT-29-shPRDX3), and control (HT-29shControl) cells, in SCID mice. Mice injected with HT-29shPRDX3 cells showed greatly reduced tumor volume and enhanced 5-FU-induced cell death compared with HT-29shControl cell-injected mice. Moreover, depletion of PRDX3 resulted in a significant reduction in liver metastasis, colon metastasis, and local invasion in an orthotopic xenograft model produced by the injection of colon CSCs into the spleen or cecum of SCID mice. Taken together, these results suggested that PRDX3 functions as an antioxidant protein, contributing to anticancer drug resistance and stemness in colon CSCs, and PRDX3 depletion reduces the distal metastasis of colon cancer via mitochondrial dysfunction.

We then investigated the mechanism underlying forkhead box M1 (FOXM1)-induced PRDX3 expression. We found that FOXM1 transcriptionally activates $P R D X 3$ and the gene encoding stem cell marker CD133 in colon CSCs. Using a bioinformatics analysis, we predicted that the human CD133 and PRDX3 promoters, would each have a FOXM1/FOXA-binding site. In particular, CD133 promoter activity was significantly reduced by the deletion of the FOXM1-binding site in a reporter assay. Chromatin immunoprecipitation assays also showed that
FOXM1 binds to the CD133 and PRDX3 promoter region that contains a FOXM1-binding site. In addition, PRDX3 and CD133 protein levels were increased by overexpression of FOXM1, resulting in an expanded $\mathrm{CD}_{133^{+}}$population and increased ATP production. Taken together, these data suggest that FOXM1-induced PRDX3 and CD133 function together to maintain the stemness of CSCs by promoting mitochondrial energy metabolism.

In conclusion, we demonstrated that highly expressed mitochondrial PRDX3 plays a critical role in maintaining mitochondrial function via the elimination of ROS produced from the OXPHOS system in CSCs (Fig. 1). This study is the first to identify PRDX3 and FOXM1 proteins as regulators of mitochondria in colon CSCs. Furthermore, our data emphasize the potential ability of combination treatment with mitochondria-targeted compounds, such as antimycin A-mediated inhibition of mitochondrial complex III or Prx3 inhibitor, and classic anticancer drugs, as a promising novel cancer therapy.

\section{ACKNOWLEDGEMENTS}

This study was supported by a grant from a Priority Research Centers Program through the National Research Foundation of Korea (NRF) funded by the Ministry of Education, Science, and Technology (2010-0020224), and a Basic Science Research Program through the National Research Foundation of Korea (NRF) funded by the Ministry of Education, Science, and Technology (2012R1A1A2041700). 